\title{
Precision measurement and compensation of optical Stark shifts for an ion-trap quantum processor
}

\author{
H. Häffner, S. Gulde, M. Riebe, G. Lancaster, C. Becher, J. Eschner, F. Schmidt-Kaler, and R. Blatt \\ Institut für Experimentalphysik, 6020 Innsbruck, Austria
}

(Dated: June 5, 2018)

\begin{abstract}
Using optical Ramsey interferometry, we precisely measure the laser-induced AC-stark shift on the $\mathrm{S}_{1 / 2}-\mathrm{D}_{5 / 2}$ "quantum bit" transition near $729 \mathrm{~nm}$ in a single trapped ${ }^{40} \mathrm{Ca}^{+}$ion. We cancel this shift using an additional laser field. This technique is of particular importance for the implementation of quantum information processing with cold trapped ions. As a simple application we measure the atomic phase evolution during a $n \times 2 \pi$ rotation of the quantum bit.
\end{abstract}

PACS numbers: $42.50 . \mathrm{Hz} 39.20 .+\mathrm{q} 32.80 . \mathrm{Qk}$

Atomic two-level systems are currently discussed extensively for quantum information processing [1, 2, 3. Typically, a qubit is encoded in an atomic transition between levels with extremely slow radiative decay: Hyperfine ground states are used as qubits which are connected via a far-off-resonant Raman transition [A]. Alternatively, the qubit is encoded in a superposition of a ground state and a long-lived metastable state [5], manipulated on a direct optical quadrupole transition. In both cases the transition matrix element between the qubit levels is small, such that relatively high laser intensities are required to drive the transition. These strong electromagnetic fields will unavoidably lead to level shifts known as the dynamical or AC-Stark shift [6].

Even though a pure two-level system does not show AC-Stark shift if driven by resonant laser radiation, in real physical situations additional energy levels exist which lead to significant AC-Stark shifts of the qubit energy levels. Since quantum algorithms manifest themselves in many-particle quantum interference, any uncontrolled phase shift induced by manipulation of the qubits complicates the implementation of these algorithms considerably and must be avoided. The interest in fast oneand two-qubit manipulations aggravates the problem [7, since strong laser fields are required. Therefore complications due to AC-Stark shifts arise in most realizations of quantum information processing with trapped ions 8, 9 . Similar problems exist in precision spectroscopy 10 and with optical clocks [11, 12].

In this paper we present a measurement which identifies different contributions to phase shifts of a qubit caused by AC-Stark level shifts. We determine the relative oscillator strengths of various contributing transitions. Finally, we show how to compensate for the ACStark shift experimentally. This compensation greatly simplifies the implementation of quantum algorithms. As an application of the compensation method we demonstrate the controlled sign change of a qubit wavefunction under rotation by $2 \pi$.

In the following we focus on the qubit transition between the $\mathrm{S}_{1 / 2}$ ground state and the metastable $\mathrm{D}_{5 / 2}$ state (lifetime $\simeq 1 \mathrm{~s}$ ) in ${ }^{40} \mathrm{Ca}^{+}$, see Fig. 1a. We drive coherently the transition between the Zeeman components $\left|\mathrm{S}_{1 / 2}, m=-\frac{1}{2}\right\rangle \equiv|\mathrm{S}\rangle$ and $\left|\mathrm{D}_{5 / 2}, m^{\prime}=-\frac{1}{2}\right\rangle \equiv$ $|\mathrm{D}\rangle$. AC-Stark shifts of these levels are induced by off-resonant quadrupole coupling to other transitions within the $\mathrm{S}_{1 / 2}-\mathrm{D}_{5 / 2}$ Zeeman manifold, as well as by off-resonant dipole coupling to other levels $\left(\mathrm{S}_{1 / 2}-\right.$ $\left.\mathrm{P}_{1 / 2}, \mathrm{~S}_{1 / 2}-\mathrm{P}_{3 / 2}, \mathrm{D}_{5 / 2}-\mathrm{P}_{3 / 2}\right)$.

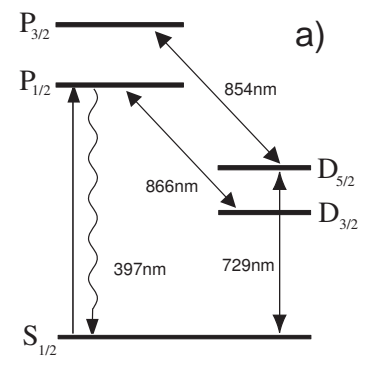

b)

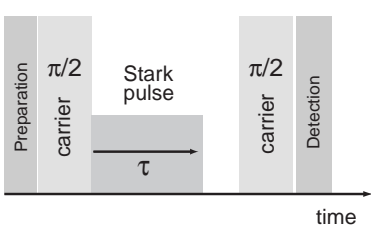

FIG. 1: a) Level scheme of $\mathrm{Ca}^{+}$ion. b) Ramsey method to detect the AC-Stark effect. The $\pi / 2$ pulses are resonant, the Stark pulse is detuned. See text for more details.

We measure the AC-Stark shift induced by a laser pulse detuned from the $|\mathrm{S}\rangle-|\mathrm{D}\rangle$ transition ("Stark pulse") with a Ramsey interference experiment 13]. For this, we enclose the Stark pulse within a pair of Ramsey $\pi / 2$ pulses resonant with the $|\mathrm{S}\rangle-|\mathrm{D}\rangle$ transition (see Fig. [1 b). Starting from the ground state $|S\rangle$, the first resonant Ramsey pulse transfers the ion to the superposition $\Psi_{1}=\{|\mathrm{S}\rangle+i|\mathrm{D}\rangle\} / \sqrt{2}$ (rotating frame). The Stark pulse shifts the levels $|S\rangle$ and $|D\rangle$ for the time $\tau$ it is applied. Since the coupling of this pulse to both levels is different, we denote the phases which are acquired as $\phi_{S}=\tau \delta_{S}$ and $\phi_{D}=\tau \delta_{D}$, respectively, and define $\Delta \phi=\phi_{D}-\phi_{S}$. Immediately before the second Ramsey pulse, the wave function is $\Psi_{2}=\left\{e^{-i \tau \delta_{S}}|\mathrm{~S}\rangle+i e^{-i \tau \delta_{D}}|\mathrm{D}\rangle\right\} / \sqrt{2}$. Now the second Ramsey pulse is applied, transferring phase information into population and yielding the state $\Psi_{3}=$ $\frac{1}{2} e^{-i \phi_{S}}\left\{\left(1-e^{-i \Delta \phi}\right)|\mathrm{S}\rangle+i\left(1+e^{-i \Delta \phi}\right)|\mathrm{D}\rangle\right\}$. Finally, $\Psi_{3}$ is projected by a measurement of the probability to find the ion in the state $|\mathrm{D}\rangle, P_{D}=\frac{1}{2}(1+\cos (\Delta \phi))$. With this method we obtain the phase shift $\Delta \phi$ induced by a Stark 
pulse applied for duration $\tau$. For a systematic measurement we vary the Stark pulse duration $\tau$ while keeping the separation of the Ramsey pulses constant. The frequency of the population variation $P_{D}(\tau)$ directly yields the Stark shift $\delta_{D}-\delta_{S} \equiv \delta_{\mathrm{AC}}$.

For the experiments, a single ${ }^{40} \mathrm{Ca}^{+}$ion is stored in a linear Paul trap made of four blades separated by $2 \mathrm{~mm}$ for radial confinement and two tips separated by $5 \mathrm{~mm}$ for axial confinement. Under typical operating conditions we observe axial and radial motional frequencies $\left(\omega_{a x}, \omega_{r}\right) / 2 \pi=(1.7,5.0) \mathrm{MHz}$. The trapped ${ }^{40} \mathrm{Ca}^{+}$ion has a single valence electron and no hyperfine structure.

We perform Doppler cooling of the ion on the $\mathrm{S}_{1 / 2}-$ $\mathrm{P}_{1 / 2}$ transition at $397 \mathrm{~nm}$. Diode lasers at $866 \mathrm{~nm}$ and $854 \mathrm{~nm}$ prevent pumping into the D states. For sideband cooling and for quantum processing [5], we excite the $\mathrm{S}_{1 / 2}$ to $\mathrm{D}_{5 / 2}$ transition with a Ti:Sapphire laser near $729 \mathrm{~nm}$ ( $\leq 100 \mathrm{~Hz}$ linewidth). With $\simeq 30 \mu \mathrm{m}$ beam waist diameter and $\simeq 50 \mathrm{~mW}$ laser power we achieve Rabi frequencies around $1 \mathrm{MHz}$, measured by driving $|\mathrm{S}\rangle$ to $|\mathrm{D}\rangle$ Rabi oscillations resonantly [4, 5]. A constant magnetic field of $2.4 \mathrm{G}$ splits the 10 Zeeman components of the $\mathrm{S}_{1 / 2}$ to $\mathrm{D}_{5 / 2}$ multiplet. The chosen geometry and polarization allow excitation of $\Delta m=0$ and \pm 2 transitions only. We detect whether a transition to $\mathrm{D}_{5 / 2}$ has occurred by applying the laser beams at $397 \mathrm{~nm}$ and $866 \mathrm{~nm}$ and monitoring the fluorescence of the ion on a photomultiplier (electron shelving technique). The internal state of the ion is discriminated with an efficiency close to $100 \%$ within $3 \mathrm{~ms}$ [阿].

The measurement cycle (total duration $20 \mathrm{~ms}$ ) consists of four consecutive steps: (i) Doppler cooling leads to low thermal vibrational states of axial and radial modes, $\left\langle n_{r}\right\rangle \approx 3$. (ii) Sideband cooling of the axial motion is performed on the $\left|\mathrm{S}_{1 / 2}, m=-\frac{1}{2}\right\rangle \leftrightarrow\left|\mathrm{D}_{5 / 2}, m^{\prime}=-\frac{5}{2}\right\rangle$ transition, leading to more than $99 \%$ ground state population. Pumping into $\left|\mathrm{S}_{1 / 2}, m=+\frac{1}{2}\right\rangle$ is counteracted by several short pulses of $\sigma^{-}$radiation at $397 \mathrm{~nm}$. (iii) Ramsey spectroscopy on the $|\mathrm{S}\rangle$ to $|\mathrm{D}\rangle$ transition, see Fig. 1]b. The $\pi / 2$ pulses have $8 \mu$ s duration and $200 \mu$ s separation. An intermediate off-resonant laser pulse (Stark pulse) with duration $\tau$, Rabi frequency $\Omega_{\mathrm{AC}}$ and detuning $\Delta_{\mathrm{AC}}$ induces a phase shift $\Delta \phi=\delta_{\mathrm{AC}} \tau$. (iv) The final state after the second Ramsey pulse is determined by electron shelving. After this, laser light at $854 \mathrm{~nm}$ pumps the ion back to the $\mathrm{S}_{1 / 2}$ ground state.

The sequence (i)-(v) is repeated 100 times to measure $P_{D}$ for each value of $\tau$ and $\Delta_{\mathrm{AC}}$. Varying $\tau$ yields data as shown in Fig. 2a. From the fit of $P_{D}$ to the data we find the AC-Stark shift $\delta_{\mathrm{AC}}$. For a given $\Delta_{\mathrm{AC}}$ each experiment was conducted twice to cancel a slow phase drift of the Ramsey pulses due to a laser frequency drift on the order of $1 \mathrm{~Hz} / \mathrm{s}$ : First the Stark pulse duration was increased with each step from $\tau=0$ to $\tau=200 \mu \mathrm{s}$, then it was decreased again. The frequency detuning $\Delta_{\mathrm{AC}}$ of the Stark pulse is varied over several Zeeman

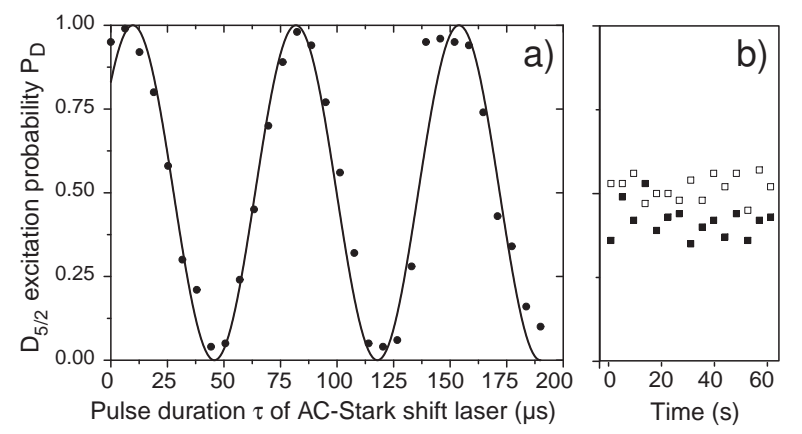

FIG. 2: (a) Ramsey pattern: The AC-Stark shift is determined as the oscillation frequency of $P_{D}(\tau)$, here $\delta_{\mathrm{AC}} / 2 \pi=$ $13.9(0.2) \mathrm{kHz}$. The phase offset is due to a small detuning of the Ramsey pulses. (b) Compensation of the AC-Stark effect: The ion is illuminated by the Stark pulse and an additional off-resonant compensation laser field which causes an equal AC-Stark shift, but with opposite sign. Data are taken alternating, with $\tau=0 \mu \mathrm{s}$ (black) and $200 \mu \mathrm{s}$ (white), see text for details. We estimate a residual $\delta_{\mathrm{AC}} / 2 \pi=0.25(3) \mathrm{kHz}$.

resonances of the $\mathrm{S}_{1 / 2}-\mathrm{D}_{5 / 2}$ manifold. Note that we extract only the modulus but not the sign of $\delta_{\mathrm{AC}}$ from the fit of $P_{D}$. The signs are attributed to the measured results according to the theoretical expectation discussed below. We determine the variation of the light intensity $I(\Delta)$ with the laser frequency detuning using a calibrated powermeter. From this we normalize the ac-Stark shift as $\delta_{\mathrm{AC}} I(\Delta) / I(0)$ to obtain the data plotted in Fig. 3 .

There are three contributions to the AC-Stark shift: In our parameter regime the largest contribution is due to the different Zeeman transitions permitted by our particular geometry $\left(m=-\frac{1}{2}\right.$ to $m^{\prime}=\frac{3}{2},-\frac{1}{2}$, and $\left.-\frac{5}{2}\right)$. The second largest contribution arises from off-resonant dipole coupling of $\mathrm{S}_{1 / 2}$ to $\mathrm{P}_{1 / 2}, \mathrm{P}_{3 / 2}$, and from $\mathrm{D}_{5 / 2}$ to $\mathrm{P}_{3 / 2}$. A third contribution is caused by motional sidebands: For a trapped ion, the Rabi frequencies on the red and blue motional sideband are given by $\Omega_{\mathrm{SB}}=\Omega \eta \sqrt{n}$ and $\Omega \eta \sqrt{n+1}$, respectively, where $\eta$ denotes the LambDicke factor and $n$ the phonon quantum number in the vibrational mode. With an ion cooled to the ground state of motion $\left(\left\langle n_{a x}\right\rangle \approx 0\right)$ and with $\eta_{a x}=0.07$, we expect $\leq 0.5 \%$ relative contribution of the blue axial sideband to the overall AC-Stark shift. The contribution of the red axial sideband vanishes. Similar reasoning holds for the radial motion with $\left\langle n_{\text {rad }}\right\rangle \approx 3$ and $\eta_{\text {rad }}=0.016$. We therefore neglect these contributions in the following.

The other relevant contributions to the AC-Stark shift can be summarized as:

$\delta_{\mathrm{AC}}=\frac{\Omega_{\mathrm{AC}}^{2}}{4}\left(2 b-\frac{a_{-5 / 2}}{\Delta_{\mathrm{AC}}-\Delta_{-5 / 2}}-2 \frac{a_{-1 / 2}}{\Delta_{\mathrm{AC}}}-\frac{a_{3 / 2}}{\Delta_{\mathrm{AC}}-\Delta_{3 / 2}}\right)$.

Here $\Omega_{\mathrm{AC}}$ denotes the Rabi frequency of the Stark laser field. The transitions $m=-\frac{1}{2} \leftrightarrow m^{\prime}=-\frac{5}{2},+\frac{3}{2}$ are at $\Delta_{-5 / 2}$ and $\Delta_{+3 / 2}$, which are $\pm(2 \pi) 8.4 \mathrm{MHz}$ in the 
experiment. An explicit calculation of the matrix elements of quadrupole transitions is given in Refs. [14, 15. The coefficients $a_{-1 / 2}, a_{-5 / 2}$ and $a_{3 / 2}$ are the squares of the relative coupling strengths. We define $a_{-1 / 2}$ to be one, since the Ramsey spectroscopy is carried out on this transition. From the laser polarization and laser axis with respect to the magnetic field axis we calculate 15 $a_{-5 / 2}=0.278$ and $a_{3 / 2}=0.0556$. The factor of 2 in the contribution of the $m=-\frac{1}{2}$ to $m^{\prime}=-\frac{1}{2}$ transition appears because the Ramsey method is applied on this transition such that the shift of both the upper and lower state is detected. From the other Zeeman components, however, only the shift of the lower state $|\mathrm{S}\rangle$ becomes apparent. The constant $b$ in Eq. (1) contains the squared relative coupling strengths to all other dipole transitions. No dependence on the laser detuning appears since the transitions are far off-resonant.

The optimum fit of Eq. (1) to the data in Fig. 3 is obtained with $a_{-5 / 2}=0.32(2), a_{+3 / 2}=0.05(2), b=$ $0.112(5) / 2 \pi(\mathrm{MHz})^{-1}$ and $\Omega_{\mathrm{AC}} / 2 \pi=357(3) \mathrm{kHz}$. We independently measured $a_{-5 / 2}=0.36(2)$ and $a_{+3 / 2}=$ 0.05 (1) with resonant Rabi oscillations. These values agree within their error margins with those obtained from the fit to the Stark shift data.

Most of the current proposals for quantum computation require that the ion is driven on the motinal sidebands. Applying a laser on the blue axial sideband of the $|\mathrm{S}\rangle \leftrightarrow|\mathrm{D}\rangle$ transition ("gate laser") at a detuning of $\Delta / 2 \pi=+1.7 \mathrm{MHz}$ results in a negative AC-Stark shift.

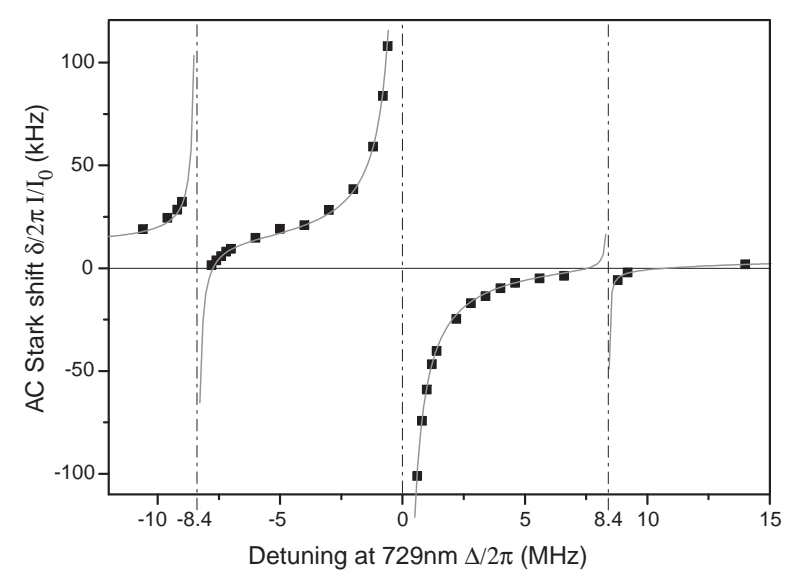

FIG. 3: The measured ac-Stark shift data (see Fig. 2a) are normalized according the measured laser power $I(\Delta) / I(0)$ which varies by about $50 \%$ over the whole tuning range of $\Delta$. This normalized data (squares) and calculated (line) Stark shift $\delta_{\mathrm{AC}}$ (Eq. ref) are plotted versus the detuning $\Delta_{\mathrm{AC}}$ of the Stark pulse from the $|\mathrm{S}\rangle-|\mathrm{D}\rangle$ resonance. The divergences are due to the $\left(m=-\frac{1}{2}\right) \leftrightarrow\left(m^{\prime}=-\frac{5}{2},-\frac{1}{2},+\frac{3}{2}\right)$ resonances (from left to right). Two data points at large detunings are not shown. They read $\delta_{\mathrm{AC}} / 2 \pi=5.88 \mathrm{kHz}$ and $8.49 \mathrm{kHz}$ for detunings $\Delta_{\mathrm{AC}} / 2 \pi=40 \mathrm{MHz}$ and $60 \mathrm{MHz}$, respectively, and are equally well described by the theoretical curve.
However, shining in a second light field at a frequency whose AC-Stark shift is positive can compensate for this unwanted phase shift. As discussed in the introduction, such an AC-Stark shift cancellation is a prerequisite for any quantum algorithm. Our method to determine the optimum setting of the compensation laser consists of the following steps: First we detune the gate laser by $\simeq 80 \mathrm{kHz}$ from the sideband resonance to avoid excitation into the D state (its AC-Stark effect however is still practically identical to that of a laser field resonant with the sideband). Then we minimize the total ACStark effect by adjusting the intensity and detuning of the compensation laser field such that the oscillations in $P_{D}$ disappear. Both light fields are generated from the output of a single Ti:Sapphire laser by driving a broadband AOM (in double pass configuration) with the two rf-frequencies simultaneously. Since both light fields are derived from the same laser, intensity fluctuations do not affect the compensation.

The accuracy to which the AC-Stark effect can be nulled is proportional to $\left(2 T_{R}\right)^{-1} S / N$, where $T_{R}$ denotes the Ramsey interrogation time (here $200 \mu \mathrm{s}$ ) and $S / N$ the signal to noise ratio of the state measurement. Integrating this measurement for long times, to improve $S / N$, is limited by the frequency drift of the laser source near $729 \mathrm{~nm}$ (typically $\leq 1 \mathrm{~Hz} / \mathrm{s}$ ), since a drift of the relative phase of the Ramsey pulses mimics a residual AC-Stark effect. To overcome this problem, we optimize the Rabi frequency and detuning of the compensation laser by alternating Ramsey experimental sequences with $\tau=0$ and $\tau \simeq 200 \mu \mathrm{s}$. Thus, a slow drift is discriminated against a residual phase shift due to imperfect compensation. Limited by the shot noise of $P_{D}(\tau)$, any AC-Stark effect can be cancelled to within $\simeq 2 \pi 30 \mathrm{~Hz}$ in $60 \mathrm{~s}$. See Fig. 2 $2 \mathrm{~b}$ for the compensation data over the course of time, each data point corresponding to 100 repetitions of the experimental sequence and a time duration of $2 \mathrm{~s}$.

As an application of the compensation method we demonstrate the sign change of a wavefunction, a simple building block frequently required in quantum algorithms. A driven spin-1/2-system transforms into its initial state only by a $4 \pi$ rotation, whereas a $2 \pi$ rotation leads to a sign change of the wavefunction. This phase shift is the central part of the Cirac-Zoller proposal [8] for quantum gates with trapped ions [16]. Similarly, Ramsey experiments on Rydberg atoms have been performed in the microwave regime, to investigate the AC-Stark shift of the electromagnetic vacuum field [17] and to perform a tunable phase gate [18].

In our experiment, the ion is first prepared in the vibrational and electronic ground state and then driven resonantly between the $|\mathrm{S}, n=0\rangle$ and $|\mathrm{D}, n=1\rangle$ state (blue axial sideband near $\Delta=+1.7 \mathrm{MHz}$ ), with the ACStark compensation laser field switched on. Fig. Ta shows the corresponding Rabi oscillations.

To measure the phase acquired during the sideband in- 


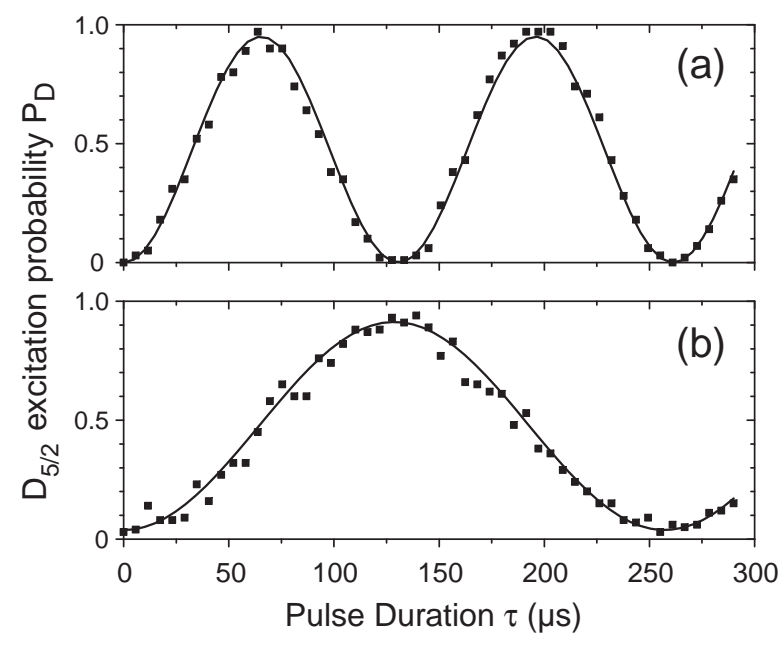

FIG. 4: (a) Resonant Rabi oscillations on the blue sideband of the $|S\rangle-|D\rangle$ transition. The period of the population oscillation is $131(1) \mu \mathrm{s}$, as found from the fit to the data. (b) Ramsey $\pi / 2$ pulses on the $|S\rangle-|D\rangle$ carrier transition enclose the Rabi flopping on the sideband. The phase of the $|S\rangle$ state is revealed to oscillate with a period of $257(2) \mu$ s. The ratio of both periods is 1.96(3) and agrees well with the expected value of 2 .

teraction we enclosed the Rabi oscillations between two carrier $(\Delta=0 \mathrm{MHz})$ Ramsey $\pi / 2$-pulses with phases 0 and $\pi$. Under the first Ramsey pulse the initial state $|S\rangle$ transforms into $(|\mathrm{S}\rangle+i|\mathrm{D}\rangle) / \sqrt{2}$, and is rotated back into $|\mathrm{S}\rangle$, in case of zero sideband interaction time. If, however, the time for the sideband interaction corresponds to a $2 \pi$ rotation on the blue sideband, the acquired phase of -1 of the population in the $|\mathrm{S}\rangle$-state results in the state $(-|\mathrm{S}\rangle+i|\mathrm{D}\rangle) / \sqrt{2}$, which is transformed to $-i|\mathrm{D}\rangle$ by the second Ramsey pulse. Only the $4 \pi$ rotation of the qubit leads back to the initial state. The experimental finding

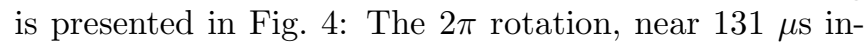
teraction time, shows up as a -1 phase shift, while after about $260 \mu$ s a full $4 \pi$ rotation is completed. In this example, the compensation laser corrected for an AC-Stark shift of $\delta_{\mathrm{AC}} \approx 2 \pi \cdot 3.1 \mathrm{kHz}$. Without compensation, this shift alone would have resulted in an additional phase of $\sim 0.82 \pi$ in $131 \mu \mathrm{s}$.

In conclusion we have precisely measured optical ACStark shifts on a single ion using an optical Ramsey interferometer. The method highlights the phase evolution an atom undergoes while it is irradiated off-resonantly with light. Measuring on a single ion removes the influence of the beam intensity profile on the measure- ment result, typically occuring with atom samples. We have also demonstrated how to compensate for the ACStark shift with a simultaneous laser pulse at another frequency. The compensation enables quantum computing on optical transitions and to detect phases of multiparticle quantum interference in a straightforward way. The quality to which the AC-Stark effect can be cancelled is proportional to the chosen Ramsey interrogation time. Therefore, it is likely that the quality of this novel phase compensation method will improve when in future longer coherence times can be realized, as necessary for a large number of quantum gate operations.

This work is supported by the Austrian 'Fonds zur Förderung der wissenschaftlichen Forschung' SFB15, by the European Commission (QSTRUCT and QI networks, ERB-FMRX-CT96-0077 and -0087, QUEST network, HPRN-CT-2000-00121, QUBITS network, IST1999-13021), and by the "Institut für Quanteninformation GmbH".

[1] Quantum Computation and Quantum Information, M. A. Nielsen and I. L. Chuang, Cambridge (2000).

[2] The Physics of Quantum Information, Springer (2000), Berlin. ed. D. Bouwmeester, A. Ekert, and A. Zeilinger.

[3] M. Sarura and V. Buzek, Journal of Mod. Opt. 49, 1593 (2002).

[4] D. Wineland et al., J. Res. Natl. Inst. Stand. Technol. 103, 259 (1998).

[5] Ch. Roos et al., Phys. Rev. Lett. 83, 4713 (1999).

[6] Atomic, Molecular, \& Optical Physics Handbook, G.W.F. Drake (ed.) (American Institute of Physics Press, Woodbury, 1996).

[7] A. Steane et al., Phys. Rev. A 62, 042305 (2000).

[8] J. I. Cirac and P. Zoller, Phys. Rev. Lett. 74, 4091 (1995).

[9] A. Sørensen and K. Mølmer, Phys. Rev. Lett. 82, 1971 (1999).

[10] D. S. Weiss B. C. Young, S. Chu, Appl. Phys. B 59, 217 (1994).

[11] K. Sengstock et al., Appl. Phys. B 59, 99 (1994).

[12] M. Niering et al., Phys. Rev. Lett. 84, 5496 (2000).

[13] N. F. Ramsey, Molecular Beams, Oxford University Press, New York 1985.

[14] D. F. V. James, Appl. Phys. B 66, 181 (1998).

[15] Ch. Roos, Doctoral Thesis, Innsbruck, Austria (2000), http://heart-c704.uibk.ac.at/papers.html.

[16] C. Monroe et al., Phys. Rev. Lett. 75, 4714 (1995).

[17] M. Brune et al., Phys. Rev. Lett. 72, 3339 (1994).

[18] A. Rauschenbeutel et al., Phys. Rev. Lett. 83, 5166 (1999). 